\title{
EchoGéo
}

28 | 2014

Police : les espaces de l'ordre, l'ordre en espace

\section{Cartographies policières : la dimension vernaculaire du contrôle territorial}

Une enquête dans la gendarmerie française

\section{Melina Germes}

\section{OpenEdition}

\section{Journals}

Édition électronique

URL : https://journals.openedition.org/echogeo/13856

DOI : 10.4000/echogeo.13856

ISSN : 1963-1197

Éditeur

Pôle de recherche pour l'organisation et la diffusion de l'information géographique (CNRS UMR 8586)

Référence électronique

Melina Germes, « Cartographies policières : la dimension vernaculaire du contrôle territorial », EchoGéo [En ligne], 28 | 2014, mis en ligne le 09 octobre 2014, consulté le 31 juillet 2021. URL : http:// journals.openedition.org/echogeo/13856 ; DOI : https://doi.org/10.4000/echogeo.13856

Ce document a été généré automatiquement le 31 juillet 2021.

EchoGéo est mis à disposition selon les termes de la licence Creative Commons Attribution - Pas d'Utilisation Commerciale - Pas de Modification 4.0 International (CC BY-NC-ND) 


\section{Cartographies policières : la dimension vernaculaire du contrôle territorial}

Une enquête dans la gendarmerie française

\section{Melina Germes}

\section{Introduction}

1 Bien que les sciences sociales francophones, dont la géographie, focalisent rarement leurs recherches sur les espaces produits par la police, l'idée que la sécurité soit une dimension fondamentale de la production de l'espace - et la police l'un de ses acteurs ne fait pas de doute, ou du moins ne devrait plus faire de doute. La géographie propre du travail policier le montre (Yarwood, 2007). De nombreuses pratiques publiques impliquent de plus en plus les institutions policières, par exemple, dans le contexte de mesures de prévention, du développement de la vidéosurveillance, et même de la mise en œuvre de projets d'urbanisme (Moreau Saint-Martin et Garnier, 2001). La police contribue à façonner l'espace social et souvent à reproduire les inégalités en pratiquant l'éviction des indésirables de l'espace public (Belina, 2003), en entretenant une forme de tension dans les quartiers réputés « sensibles » (Jobard, 2005).

2 Cependant, dans le contexte contemporain de la géographie francophone il est nécessaire de construire l'objet de recherche "police»; tout comme de montrer la pertinence de la contribution de problématiques géographiques aux recherches pluridisciplinaires sur la police. Deux ressources sont fondamentales pour ce projet: premièrement, l'interdisciplinarité, grâce aux outils et aux discussions issues de la sociologie et des sciences politiques (voir, entre autres: Brodeur et Monjardet, 2003 ; Mucchielli et Robert, 2002) ; deuxièmement, le recours aux approches géographiques de la police développées dans les sciences sociales anglophones (Evans et al., 1992 ; Fyfe, 1991 ; Herbert, 1996, 2003) et germanophones (Belina, 2006 ; Eick et al., 2007 ; Glasze et al., 2005). Au sein des organisations toujours plus nombreuses qui contribuent à la 
"sécurité ", la place de la police ${ }^{1}$ est fondamentale. Elle est depuis longtemps considérée comme le bras armé de l'État (au sens de pouvoirs publics, dans leur diversité) pour la sécurité intérieure : elle exécute (c'est-à-dire interprète et applique) les décisions prises par les autorités politiques et administratives mandataires. Cette place évolue avec le changement organisationnel constitué par l'établissement de nouveaux régimes néolibéraux de sécurité (Eick et al., 2007), marqués par une culture du contrôle (Garland, 2001) et l'idée d'un "tournant punitif» global consommé également en France (Ferret et Mouhanna, 2005 ; Mucchielli, 2008a).

Il m'apparaît de plus nécessaire d'ancrer cette construction d'un objet géographique dans le contexte d'une problématisation critique de l'espace - c'est-à-dire dans les discussions théoriques qui envisagent l'espace comme un enjeu de luttes et de contestations, tant dans le monde germanophone (Belina, 2008, 2013) que francophone (Bautes et Marie Dit Chirot, 2012 ; Morange et Calbérac, 2012). L'espace est à la fois une matérialisation des rapports de force et des inégalités, l'objet d'appropriations contradictoires voire conflictuelles et une représentation réductrice de la complexité $\mathrm{du}$ social. On peut ainsi faire le postulat que l'espace est l'instrument d'une coercition que la police met en œuvre.

4 Ce texte contribue à une géographie de la police en ce qu'il s'interroge sur la production de l'espace par la police grâce à l'étude du travail que constitue la production, l'utilisation de cartes et l'ensemble des pratiques cartographiques. Ces dernières - l'ensemble des moments auxquels les cartes sont lues, réalisées, transmises, discutées - m'intéressent à trois titres. Premièrement, ce sont les moments où les représentations (idéelles) de l'espace sont réaffirmées et réévaluées en lien étroit avec les pratiques sociales de l'espace, qui précèdent et qui lui succèdent. Deuxièmement, ce sont des moments charnières de communication entre des acteurs dotés de différents rôles au sein d'une institution complexe et très différenciée (et particulièrement comme charnière entre le travail sur le papier et sur le terrain, cf. Mensching, 2008) contrairement aux situations en poste de police, en patrouille ou en intervention. Troisièmement, ils sont au cœur du problème de la modernisation de l'action publique : dans la police comme ailleurs, l'injonction d'un recours à l'évaluation et à l'expertise transforme les pratiques quotidiennes de la statistique mais aussi de l'intervention (Didier, 2011b; Mucchielli, 2008b). La cartographie est ainsi l'un des instruments de l'action publique qui semble répondre à la demande néolibérale d'expertise (Lascoumes et Le Galès, 2004).

5 La dernière et principale raison de mon intérêt pour les pratiques cartographiques policières réside dans la conception des cartes comme instrument de pouvoir - en tant que moyen de l'exercice d'une emprise concrète sur l'espace et de configuration des représentations du monde. Les cartes seraient un instrument idéal de la production de territoires de souveraineté. Que deviennent les cartes dans les mains de la police? Servent-elles à produire des espaces appropriés et contrôlés, de véritables territoires policiers ? À quelles pratiques cartographiques sont attribuées quelles fonctions? L'enquête qui suit aborde cette question à partir d'une enquête dans la gendarmerie française ${ }^{2}$.

6 Nombreux sont les travaux sur les cartographies de la criminalité (dans la police, mais aussi dans les médias, sur internet, faites par des sociétés de transport ou d'habitat social etc.) qui utilisent les nouveautés des Systèmes d'Information Géographique (SIG) et les potentialités du Web 2.0. Or, ces travaux ignorent ou sous-estiment deux 
phénomènes fondamentaux qui sont omniprésents sur le terrain de mon enquête dans la gendarmerie française: premièrement, le fait que la police produit et manipule d'autres cartes que celles dites "de la criminalité », et ce bien avant la révolution numérique; deuxièmement, le fait que les outils de crime mapping ${ }^{3}$ nécessitent des formations, des équipements informatiques mais aussi une adaptation de l'institution et une reconfiguration des modalités du travail (Manning, 2001). Ces deux phénomènes questionnent le postulat d'une pratique cartographique disposant des outils techniques, des informations et des formations nécessaires à son efficacité, d'une pratique qui pour les uns contribue à la sécurisation nécessaire du territoire, qui pour les autres sert au contrôle abusif de la population. Même les travaux qui puisent leurs arguments et méthodes dans la cartographie critique et mettent en cause la légitimité des cartes de la criminalité ne perçoivent pas les pratiques cartographiques comme des éléments d'une routine pourtant ancienne ni le sous-équipement de nombreuses polices. Ceci conduit, dans la première partie, à choisir une approche centrée sur l'observation des pratiques.

7 La deuxième partie analyse les pratiques cartographiques dans la gendarmerie française par rapport au problème que constitue le constat de compétences et de moyens institutionnels bien en-deçà des exigences d'une expertise géocriminologique. Les pratiques cartographiques sont omniprésentes dans le travail de gendarmerie à plusieurs échelons et dans plusieurs métiers. Elles sont ambivalentes, entre routine et désir d'innovation, entre illusion cartographique et désillusion technologique. L'observation ethnographique des incertitudes et des déboires face aux pratiques cartographiques déplace la question d'un pouvoir intrinsèque aux cartes.

La troisième partie propose une typologie des pratiques cartographiques à partir des catégories vernaculaires du travail de gendarmerie : la cartographie administrative, la cartographie d'analyse, la cartographie opérationnelle. Les images, fixes ou animées, du corpus de documents recueillis sont étudiées telles qu'elles sont mises en œuvre dans les processus de travail, afin de mettre en évidence comment le travail de représentation cartographique contribue à trois projets territoriaux - à trois tentatives d'appropriation de l'espace.

\section{Cartes de la criminalité et cartographies critiques : reformulation d'une approche}

\section{Cartographie du crime dans la police}

9 L'étude des cartographies policières est pour l'instant limitée dans la littérature au crime mapping, en laissant de côté les autres cartographies (des zones de compétences, pour la planification d'opérations, etc.). La pratique de la cartographie du crime se situe à la convergence entre les concepts de la criminologie environnementale (ou géocriminologie) et de l'ingénierie cartographique. Les travaux fondateurs de Patricia et Paul Brantingham (1991 [1981]) reposent sur l'idée que «l'espace et le lieu (place) déterminent le moment, le lieu et le caractère des crimes » et étudient ainsi le motif (pattern) et la géométrie des crimes et délits. Les efforts des nombreux auteurs contribuant à la criminologie environnementale se déploient dans deux directions : premièrement, un travail sur les fondements conceptuels reliant explications criminologiques et espace (Weisburd et al., 2009); deuxièmement, un travail sur les 
applications en termes de prévention de la criminalité et d'intervention policière (Brantingham et al., 2010). La cartographie de la criminalité fait alors partie du métier «d'analyste criminel » auquel sont formés des étudiants en criminologie et ainsi d'une forme particulière d'organisation du travail policier dirigée par l'expertise et l'information, basée sur l'établissement de bases de données de l'activité policière telles CompStat (à propos de son application à Paris: Didier, 2011a). Elle est utilisée essentiellement aux États-Unis, Canada, Royaume-Uni, Australie et Afrique du Sud, par des polices métropolitaines et elle est implantée en général dans le cadre d'un plus vaste changement, où moyens technologiques, organisation du travail et son management sont interdépendants (Chan, 2004a). Elle présuppose donc une adaptation institutionnelle (Mazerolle et al., 1997; Ratcliffe, 2002), comme d'autres outils de surveillance (Purenne et Wuilleumier, 2011). Des changements comparables en termes de nouveau management ont eu lieu en France, où quelques experts réclament l'introduction du crime mapping encore absent (Camilleri et Lazerges, 1992; Besson, 2005 ; Cunty et al., 2007).

Les crime maps prennent des formes très variées qui reprennent les multiples potentialités de l'analyse spatiale avec des représentations « de la criminalité » en hot spots (nuage de points), en densité selon les grilles ou par circonscription (et ce, à partir des statistiques liées à l'activité policière), le geographic profiling (dans le but de déterminer le lieu d'habitation d'un coupable (Rossmo et Rombouts, 2008), la prédiction du lieu du prochain crime (Groff et La Vigne, 2002), les tentatives de modélisation de " modes opératoires", les heat map (cartes dites de chaleur ou d'interpolation), etc. Les nombreuses publications qui présentent des études de cas techniquement raffinées sont les résultats d'expérimentations plutôt exceptionnelles (réalisées par des spécialistes dans le cadre de projets limités dans le temps et dotés de moyens importants, voir les ouvrages collectifs : Chainey et Tompson, 2008; Weisburd et al., 2009) et rendent très peu compte de la routine des usages. Les cartographies de la criminalité sont des objets de choix pour la cartographie critique, qui questionnent leur pertinence sous plusieurs aspects.

\section{Critique des cartes (du crime)}

11 Une première approche critique interroge les cartes (en général) comme les instruments stratégiques de la conquête d'un territoire et de gouvernement des peuples. Elles sont des instruments idéologiques de la légitimation de l'État (Neocleous, 2003). Elles sont validées par des méthodes réputées scientifiques. Elles naturalisent les frontières et transmettent des représentations d'un corps politique territorialisé et approprié : des cartes dessinant le paysage de la renaissance aux mappemondes arabes, chinoises et européennes, en passant par les cartes topographiques à partir du XVIII ${ }^{e}$ et $\mathrm{XIX}^{\mathrm{e}}$ siècles, jusqu'aux cartes choroplèthes des «statistiques morales» (Guerry, 1833) puis les statistiques publiques et enfin les cartographies élaborées de l'aménagement des territoires.

12 Au contraire des promoteurs de la cartographie de la criminalité, qui affirment la scientificité, la technicité et l'efficacité de cette dernière, ses détracteurs questionnent premièrement l'idéologie politique qui sous-tend le discours scientifique de la géocriminologie (Belina, 2000; Peet, 1975); deuxièmement les simplifications, réductions et abstractions issues de la représentation graphique - toute technique 
qu'elle soit (Beaude, 2009; Belina, 2009). La mise en œuvre d'analyses à partir des statistiques de l'activité policière conduit finalement à intensifier le contrôle policier sur les espaces, les personnes et les délits déjà les plus intensément contrôlés, renforçant ainsi la discrimination policière.

Ces critiques, toutes pertinentes qu'elles soient, prennent comme objet des cartographies telles qu'elles sont pensées, conçues, élaborées par des experts. Elles ne prennent pas en considération d'une part, les pratiques cartographiques dans le quotidien du travail policier ni d'autre part, les cartographies (pourtant existantes depuis longtemps) dans des institutions n'ayant pas consommé, ou ayant engagé d'une autre manière les transformations organisationnelles évoquées ci-dessus. Les discussions sur le crime mapping, qu'elles soient critiques ou emphatiques, souscrivent le plus souvent au postulat d'une technicité de la pratique (par définition même de l'objet crime mapping); de la même manière, de nombreuses études sur la vidéosurveillance sont basées sur le postulat d'un "déterminisme technologique " qu'une approche ethnographique du travail des surveillants remet en question (Norris et Armstrong, 1999 ; voir aussi Le Goff, 2013). Si de nombreux arguments et concepts utilisés dans les approches critiques de la cartographie de la criminalité sont opératoires pour la cartographie en gendarmerie, ils ne permettent pas de penser la complexité des pratiques cartographiques, confrontées à (mais non déterminées par) la technologie.

Le déplacement de l'objet de recherche (au-delà du seul crime mapping, vers les cartographies policières) et le décentrement du terrain (sur la gendarmerie française, en marge des hauts lieux du crime mapping) sont accompagnés d'une évolution du cadre méthodologique et théorique de la recherche. Le but de la recherche est d'étudier le travail cartographique policier par rapport à l'ambition du contrôle du territoire tout en tenant compte des cultures professionnelles policières, de l'environnement technologique et des significations attribuées par les acteurs à leurs pratiques.

\section{Une analyse critique des pratiques cartographiques}

Une deuxième approche de cartographie critique a pris au sérieux l'idée d'une contextualisation de l'objet carte dans des pratiques sociales complexes. La carte en soi n'est rien sans les pratiques qui la produisent, la modifient et l'interprètent (Perkins, 2008 ; Pickles, 2004) ; elle n'est pas un simple discours graphique, mais elle fait l'objet d'interactions (Del Casino et Hanna, 2005). Cette évolution paradigmatique est accélérée par la généralisation des moyens informatiques de production et de diffusion de documents (papier ou électroniques), d'images (fixes ou interactives), à la multiplication des plates-formes de cartographie, à la facilité de circulation électronique des images et à la cartographie participative (Crampton, 2009 ; Joliveau et al., 2013). Une grande partie des cartes ne sont plus des images-documents, mais des images interactives, électroniques, mouvantes, le simple reflet de données en évolution permanente. Par ailleurs, la reconnaissance croissante et l'émergence de nouveaux acteurs ayant recours à la cartographie - groupes indigènes, artistes, mouvements sociaux - complexifient les analyses sur la production, la diffusion, la mise en œuvre et la lecture des cartes. Celles-ci peuvent servir d'autres fins à l'aide de moyens de plus en plus divers, voire même faire partie de projets émancipateurs (Herb et al., 2009). La mouvance des images et la malléabilité des significations sont ainsi assumées. 
16 La complexification des objets d'étude a permis de repositionner la dimension critique de la cartographie critique : les usages, les intentions, les interprétations, les pratiques cartographiques (et non plus les «seuls » documents) sont questionnés certes par rapport à des enjeux de domination et d'émancipation, mais aussi d'instrumentalisation et de détournement.

Quelle en est la conséquence pour l'étude des cartographies policières ? Contrairement à de nombreux «nouveaux objets » étudiés dans le cadre de cette deuxième approche de cartographie critique (comme la cartographie participative, les contre-cartographies réalisées par des mouvements sociaux ou des artistes contestataires), la spécificité des cartographies policières est de rester une pratique orthodoxe au sein d'une institution étatique hégémonique. Les pratiques cartographiques exposées ci-dessous travaillent au plus près de la violence d'État et de la contrainte policière, elles font partie d'un projet de gouvernement dont les répercussions s'étendent du management des institutions policières à la surveillance de la population. Elles doivent pourtant aussi être comprises comme des manières de faire (en référence au concept de Michel de Certeau) car elles ne répondent pas forcément à des protocoles unifiés, centralisés et contrôlés qui produiraient des pratiques homogènes. Les enquêtes montrent que l'appareil d'État ne dispose pas nécessairement des outils techniques et des savoir-faire nécessaires à la pratique cartographique - et à ses prétentions territoriales via les cartes. Même si elles ont lieu en contexte professionnel, les pratiques cartographiques policières relèvent en partie de manières de faire autodidactes ou dilettantes : ce qui correspond à l'évolution des pratiques cartographiques dans le contexte d'un large recours aux possibilités du geoweb dans la société (Crampton, 2009).

Les pratiques cartographiques contribuent certes en tant que «travail de représentation » à des constructions territoriales particulières et ainsi à l'exercice de la coercition et de la contrainte. Ces pratiques sont étroitement encadrées par une culture professionnelle $e^{4}$, ayant une historicité particulière et un système de significations spécifiques: la recherche se focalise ainsi sur les manières de parler et de faire de ce monde professionnel particulier, afin d'en comprendre les catégories, afin de restituer les pratiques dans le contexte d'intelligibilité des acteurs et de leurs raisonnements. C'est la dimension vernaculaire de la pratique cartographique qui m'intéresse ici, selon deux significations qui entretiennent des relations paradoxales avec l'expertise : tout d'abord, selon la signification d'une appropriation vernaculaire des outils technologiques tels le Web 2.0 et la cartographie par des acteurs en contexte professionnel sans norme professionnelle d'usage de ces outils (voir Howard, 2008). Deuxièmement, au sens où la culture professionnelle de la gendarmerie conduit à l'établissement d'un savoir géographique vernaculaire (Collignon, 2005). Tant les pratiques de l'outil cartographique que les savoirs géographiques mobilisés par la gendarmerie relèvent de cet univers professionnel, loin du monde savant des cartographes d'une part et des géographes ou sociologues d'autre part.

\section{Méthodologie de l'enquête de terrain}

Les enquêtes de terrain suivantes ont pu être réalisées en 2013 dans le cadre d'un projet de recherche collectif bénéficiant d'accès à de nombreux interlocuteurs au sein de la gendarmerie française grâce à un partenariat avec l'École des officiers de la Gendarmerie Nationale (EOGN) et à des autorisations d'enquêtes de la Direction 
Générale de la Gendarmerie Nationale (DGGN). Le petit groupe de deux ou trois chercheurs était présenté aux interlocuteurs respectifs par un supérieur hiérarchique. Le motif de notre visite était le recensement des bonnes pratiques dans le cadre du partenariat avec l'école d'officiers afin de contribuer à la formation continue, ce qui explique la grande disponibilité et la confiance de nos interlocuteurs.

La recherche s'est déroulée à différents échelons et dans différents milieux et métiers de la gendarmerie ${ }^{5}$ : au sein d'institutions nationales (offices et Écoles), de groupements de gendarmerie (au niveau départemental) et au sein de brigades locales (niveau pluri-communal). Cet article aborde ainsi essentiellement des situations de travail en bureau'.

21 L'enquête s'est déroulée sous la forme d'immersions de courte durée (d'une journée à deux semaines selon les lieux). Je me présentais comme la géographe de l'équipe de sciences sociales dans le but de justifier mon intérêt pour les pratiques cartographiques. J'ai pu mener 35 entretiens semi-collectifs, assister à des démonstrations d'usage (environ 20 courtes séquences de travail), et voir de nombreux documents dont seulement une partie a été collectée (soit 22) ${ }^{7}$. Au cours d'entretiens sur les modalités du travail et les pratiques professionnelles (parfois seule, parfois en groupe), j'ai pu observer de nombreuses cartes, rassembler un corpus de documents (imprimés ou électroniques), et parfois observer leur utilisation, sans dissocier les documents des récits qui les entourent.

\section{Petite ethnographie d'une pratique vernaculaire de la cartographie dans la gendarmerie française}

L'objet "pratique cartographique " est une construction conceptuelle issue d'une démarche de sciences sociales qui ne correspond dans la gendarmerie française contemporaine ni à un métier, ni à un service, ni à un savoir-faire explicite. Il désigne une multitude d'objets ou d'éléments, épars dans travail quotidien de police dont ils ne sont qu'un moment.

\section{Traditions et évolutions cartographiques}

Les gendarmes se présentent volontiers comme des généralistes de la sécurité et des experts du territoire local: le rôle des gendarmes de brigade est, dans l'idéal, de connaitre «leur» territoire, routes, chemins et lieux dits sur le bout des doigts, de connaître tant « leur " population que tout ce qu'il se passe localement; finalement de connaître tellement bien le territoire au point de ne plus avoir besoin de carte pour s'orienter. Relativement peu présente sur le "terrain ", la carte renvoie plutôt aux situations de commandement en cas de crise ou bien au travail de bureau quotidien : le mot « carte " évoque pour mes interlocuteurs en premier lieu les cartes topographiques au $50.000^{\mathrm{e}}$ les anciennes cartes d'État-Major, qui font partie du volet militaire de la formation et qui concernent en priorité les officiers, c'est-à-dire les échelons moyens de la hiérarchie. L'apprentissage traditionnel de la lecture militaire d'un paysage passe par la pratique de courses d'orientation et l'élaboration de plans de déploiement et de repli en situation. Cette pratique militaire de la carte d'État-major qui pourrait paraître 
désuète est néanmoins un élément fondamental de l'identité professionnelle en gendarmerie.

Cet héritage, élément fondamental d'une culture professionnelle propre à la gendarmerie, fait face aux changements majeurs du travail de police évoqués précédemment. Concrètement, ces changements sont caractérisés par l'évolution fondamentale des outils de travail, leur informatisation croissante en lien avec la constitution de bases de données normalisées, centralisées, contrôlées. Depuis 2010, plusieurs bases de données centralisées ont été ainsi introduites dans la gendarmerie, pour des activités très différentes, entre autres: la gestion opérationnelle et la remontée de l'information (BDSP) et la gestion de l'information de sécurité publique, la saisie et le traitement des enquêtes et procédures judiciaires (LRPGN) complètent la base de données des statistiques de gendarmerie selon l'état 4001 (Infocentre). Infoncentre comme BDSP disposent de modules cartographiques.

L'évolution des outils, de la culture professionnelle et des savoir-faire de la gendarmerie permet d'émettre l'hypothèse que la cartographie experte de la criminalité est (de plus en plus) implantée dans la gendarmerie française - comme elle l'est dans d'autres institutions. Or, la gendarmerie française contemporaine n'est pas confrontée de la même manière que les polices nord-américaines ${ }^{8}$ à ces changements en termes de management et de procédures de travail; il ne s'agit pas d'un simple transfert. Les doctrines évoluent certes dans le sens d'un tournant punitif - ou d'une politique sécuritaire - mais les multiples adaptations locales de la doctrine et les quelques résistances dans l'institution s'expriment autrement. Si une place de plus en plus importante est faite à l'analyse, il ne s'agit cependant pas d'un métier à part entière bénéficiant d'une formation spécifique. 
Illustration 1 - La carte murale du CORG, Groupement de la Gironde

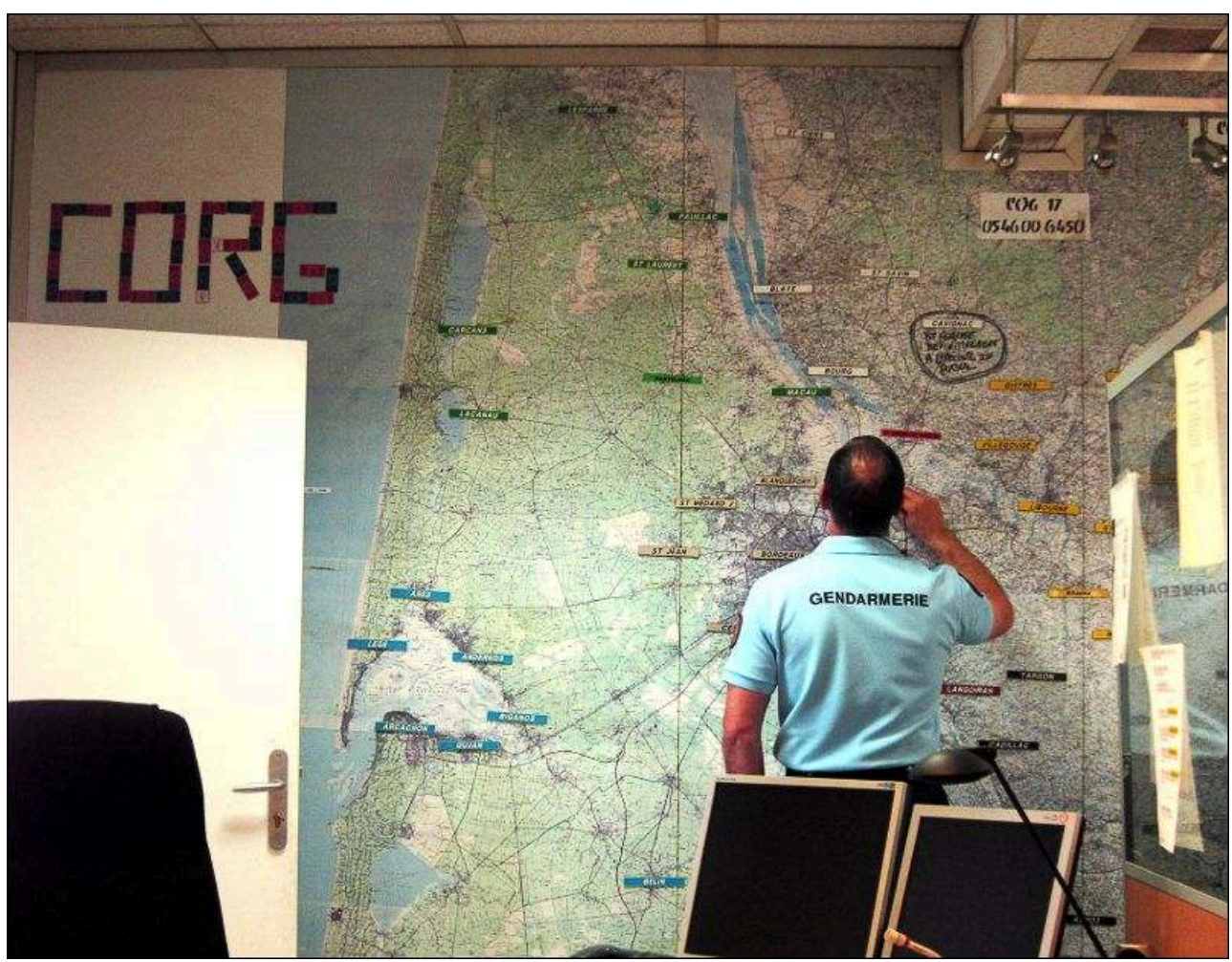

Suite à un flagrant délit, le CORG synchronise les interventions de différentes unités véhiculées pour intercepter le suspect en fuite (plan épervier). Or, ce jour-là, la base de données nationale consacrée, BDSP, ne fonctionne pas : l'adjudant-chef a dû avoir recours à la cartographie murale. II s'agit d'un collage mural de cartes IGN montées sur un support métallique et plastifiées, équipé d'aimants (noms de communes, figurés de véhicules), réalisé à l'époque grâce à l'aide matérielle d'un autre service public

Auteur: M. Germes, 2013.

\section{L'omniprésence des cartes}

Les cartes omniprésentes dans le travail de gendarmerie sont mises en œuvre dans des démarches très différentes: sur des diapositives pour présenter au public ou aux partenaires publics l'espace de compétence et les derniers chiffres ou dispositifs; en affichage récurrent sur les lieux de travail; comme illustrations dans des rapports confiés au supérieur hiérarchique ; en tant que document d'analyse d'un phénomène ; comme ordre de mission pour une intervention à l'extérieur, etc. Elles sont consultées sur écran ou sur papier, imprimées, annotées.

Le recours aux cartes est, pour les acteurs, essentiellement instrumental. Même les grandes cartes affichées sur les murs des bureaux des supérieurs hiérarchiques ne sont pas appréciées pour une valeur symbolique ni décorative, comme cela peut être le cas de nombreuses mappemondes ostentatoires dans d'autres contextes professionnels.

\section{Un équipement succinct}

Contrairement à l'hypothèse précédente, il n'y a ni grande révolution de la cartographie de la criminalité impliquant SIG et expertise géocriminelle, ni persistance 
désuète d'un recours aux cartes topographiques. L'équipement cartographique très succinct évolue à la fois par le centre et par les marges.

Les nouvelles bases de données centralisées qui viennent d'être mises en place dans la gendarmerie nationale avec de très lourds efforts de formation offrent pourtant des modules cartographiques. BDSP (Base de Données de Sécurité Publique de la Gendarmerie Nationale, destinée à l'intervention et au renseignement) permet la géolocalisation en temps réel de tous les véhicules de la GN disposant d'une identification par GPS, et la géolocalisation des événements enregistrés par les centres d'appel d'urgence (cf. illustration 7). Infocentre (destiné à la compilation des statistiques de police) permet la représentation par points de délits et crimes classifiés selon l'état 4001 sur une période donnée et dans une circonscription, à la condition expresse (et rarement remplie) qu'une adresse normalisée ait été saisie. Ces modules cartographiques permettent des ébauches de localisation d'évènements souvent mal désignés ou mal localisés, à la condition de se contenter d'une grande échelle (voir paragraphe sur les polygones de la cartographie administrative).

Sur le terrain des brigades, des compagnies, des groupements de gendarmerie et de certains services centraux, les acteurs mobilisent donc d'autres ressources pour leurs pratiques cartographiques. Une pratique devenue obsolète consiste à compléter par des ajouts avec un logiciel de dessin (Paint p. ex.) un extrait de carte topographique ou routière numérisé et. Depuis, les cartes sont réalisées à partir des outils de la cartographie 2.0 - Google Maps, Viamichelin, Open Street Map. Les copies d'écran des plans ou des images aériennes sont éventuellement complétées à l'aide d'un logiciel de dessin ou le détournement d'un traitement de texte. Plus rarement, des cartes sont réalisées avec le logiciel de cartographie MapPoint, qui permet une analyse succincte de données géolocalisées. Ce travail est parfois réalisé sur des ordinateurs portables privés, branchés à côté de l'ordinateur de service, avec des logiciels tantôt gratuits tantôt acquis sur les deniers personnels. La plupart des documents se présente comme un enchevêtrement de dessins assistés par ordinateur et de géolocalisation. Ce sont les ressources individuelles - le savoir-faire, la débrouillardise, l'autodidactisme - qui jouent un rôle fondamental dans ces pratiques cartographiques, beaucoup plus que les ressources mises à disposition par l'institution.

31 Les cartes apparaissent ainsi à la fois comme un élément culturel essentiel et récurrent de la routine professionnelle mais ne sont pas profondément marquées par l'évolution des méthodes de travail qui ont eu lieu avec d'autres outils statistiques et d'analyse.

\section{Une culture professionnelle sans expertise}

32 Cela explique probablement que mes interlocuteurs, sur le terrain, soient rétifs et peu enthousiastes à l'idée de me présenter les cartes qu'ils ont eux-mêmes réalisées. Ils en sont peu satisfaits et insistent sur le fait qu'il s'agit «seulement d'une tentative». Ils espèrent remplir les attentes de l'institution (en termes d'élucidation et d'explication par les cartes). Ils sont motivés par l'existence d'un crime mapping, et ils trouvent en même temps des ressources dans les instruments accessibles au grand public qui s'approprie de plus en plus d'outils cartographiques. Cependant, leurs formations initiales ne les dotent que rarement des savoirs et savoir-faire nécessaires. Les apprentissages en matière de cartographie et d'utilisation des logiciels sont ainsi en général autodidactes et ne sont pas accompagnés d'un apprentissage criminologique - 
ni en termes d'expertise géocriminologique, ni en termes de déconstruction de cette dernière par les sciences sociales. Les manières de faire des cartes sont des petits arrangements avec les ressources disponibles, marqués par des tentatives locales d'innovation à tâtons et restent des initiatives individuelles.

Les cartographies produites peuvent être passées au crible de la critique experte - de la même manière que des productions cartographiques non professionnelles. Nombre de documents consultés sont problématiques selon les critères des cartographes experts par rapport à l'utilité qui leur est attribuée. La critique cartographique relèverait comme problèmes principaux l'arbitraire des discrétisations, le choix non pertinent de figurés et l'illisibilité des représentations élaborées (voir sur ce point l'agacement de Field, 2011). La critique des données relèverait surtout le fait que les sources ne rendent compte ni de la criminalité, ni même de l'activité de police : les bases de données sont mal remplies (Didier, 2011b), les recensements sont incomplets et faussent ainsi même une « cartographie des statistiques de l'activité policière ». Même si certains gendarmes qui manipulent les cartes sont conscients que les réalisations ne correspondent pas à leur projet, ils attribuent la responsabilité à l'absence de logiciel, d'outil technique adapté et accordent trop peu d'importance aux éléments criminologiques et cartographiques liés au sens de ce qu'ils représentent et à la manière de représenter.

Malgré les problèmes exposés, les cartes sont produites et utilisées dans la gendarmerie française et fonctionnent comme un instrument efficace de communication et de partage de représentations. Elles montrent, elles parlent, elles font sens : elles illustrent des propos et servent de support relativement malléable à des discours sur l'espace. Les énoncés qui entourent les cartes et les font vivre font de multiples références à un discours sur l'espace, à «ce qu'on ne voit pas sur la carte » mais que l'on sait, par ailleurs. Le moment cartographique est celui où l'acteur met en œuvre un savoir géographique justifié par son expérience professionnelle et fortement adossé aux représentations dominantes. L'interaction des gendarmes avec les cartes montre qu'elles sont rarement utilisées pour apporter une nouvelle représentation, une nouvelle interprétation mais au contraire pour apporter la confirmation d'une représentation existante, pour en être le support visuel, la mise en évidence. La visualité des cartes fascine les gendarmes autant que d'autres usagers ( $\mathrm{du}$ grand public aux professionnels); mais plus encore lorsqu'il s'agit de cartographier la délinquance et la criminalité, la culture visuelle du crime fascine (Wallace, 2009).

En l'absence de normes professionnelles explicites et d'un savoir-faire spécifique, ces pratiques cartographiques hybrides sont pourtant encadrées par d'autres normes professionnelles implicites et consolidées. Ces autres normes prennent place dans des routines de travail spécifiques, dans une culture professionnelle particulière que la troisième partie de ce texte déchiffre en détail.

\section{Dessins du projet territorial}

Cette troisième partie propose de poursuivre le questionnement sur la dimension vernaculaire des pratiques cartographiques et sur la constitution d'une norme professionnelle implicite en montrant, grâce à des exemples de travail cartographique, comment ces pratiques contribuent à la production institutionnelle d'un territoire policier - ou, plus exactement, quel rôle elles jouent dans l'accomplissement d'un projet territorial. 

concept géographique controversé, discuté par les géographies politiques et sociales des mondes francophones et anglophones ${ }^{9}$, qui est parfois étroitement associé à la cartographie ; il s'agit d'autre part d'une notion fondamentale et quotidienne du travail policier (qui s'impose aux chercheurs problématisant l'espace policier : Herbert, 1997). Il s'agit d'une catégorie apparemment évidente pour penser le travail de police - et ses territoires de compétence ou ses territoires à sécuriser ; et d'une notion indispensable à la compréhension de l'évolution de la surveillance et du contrôle par l'État (Herbert, 1999). Je propose de considérer avec le terme territoire un travail quotidien d'appropriation au nom de l'État (voir la conception du territoire comme effet chez Painter, 2010), en fonction d'un projet territorial propre à chaque institution, ses missions et ses moyens. Je ne considère pas que les cartes suffisent à produire un territoire policier, pas plus qu'elles ne l'illustrent ou le traduisent. Elles constituent un travail de représentation, au sein d'une institution, selon des procédures de travail spécifiques et elles contribuent ainsi à la mise en œuvre d'un projet territorial. Les notions de «travail » d'appropriation et de "projet » territorial n'impliquent pas le succès, la réussite, l'accomplissement - mais seulement la recherche d'une emprise, d'un contrôle, d'une certaine souveraineté sur l'espace.

L'étude du travail cartographique se concentre à la fois sur les processus de production concrète des cartes et sur leur utilisation, et sur les significations qui leurs sont attribuées. Les cartes ne sont pas considérées «en soi " mais resituées dans leur environnement : le texte écrit et oral, les supports (papier, électronique) ; les formes de visualisation (listes, tableaux, schémas relationnels) et les interactions et circulations dont elles font l'objet. J'utilise la notion d'abstraction pour analyser le travail cartographique (cf. Belina, 2009 et en conclusion ci-dessous). Trois catégories vernaculaires et ainsi trois missions du travail de gendarmerie que sont le travail " administratif », "d'analyse ", " opérationnel », permettent de distinguer trois types de cartes - qui, par ailleurs, ne renouvellent pas les registres connus de la cartographie.

\section{Les polygones de la cartographie administrative}

Dans le vocabulaire de gendarmerie, le terme «administratif» désigne le travail qui concerne directement l'organisation de l'institution - le partage des rôles et des domaines de compétence, l'emboîtement spatial et hiérarchique des responsabilités. La représentation du maillage territorial est un élément central de la cartographie administrative.

\section{Cartographie administrative des territoires de compétence}

Le premier exemple regroupe des cartes murales affichées dans les bureaux des échelons médians : les illustrations 2 et 3 montrent deux représentations du territoire de compétence. 
Illustration 2 - Le Groupement du Rhône, Gendarmerie Nationale

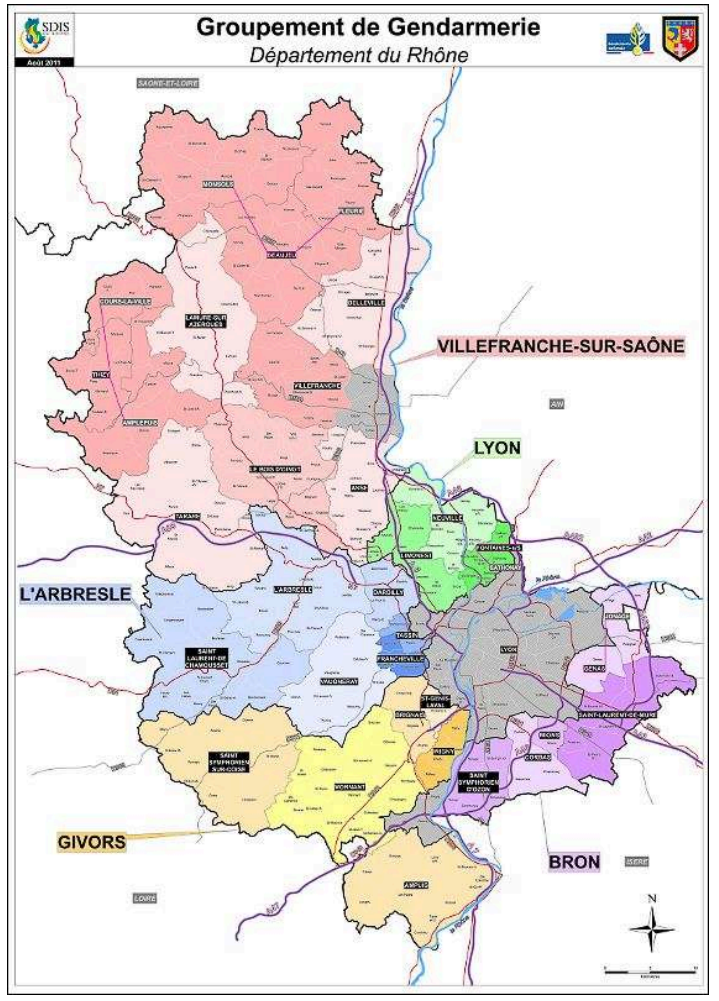

Document PDF imprimé au format A1, affiché dans plusieurs bureaux du Groupement du Rhône à Lyon (Commandant, CORG, BRDIJ...).

Réalisation : SDIS (Service Départemental Incendie Secours), 2011.

41 La première (ci-dessus) met l'accent sur l'imbrication des « unités territoriales », sur le pavage des compétences de la gendarmerie départementale; la seconde (ci-dessous) met l'accent sur la topographie et sur la localisation des brigades. 


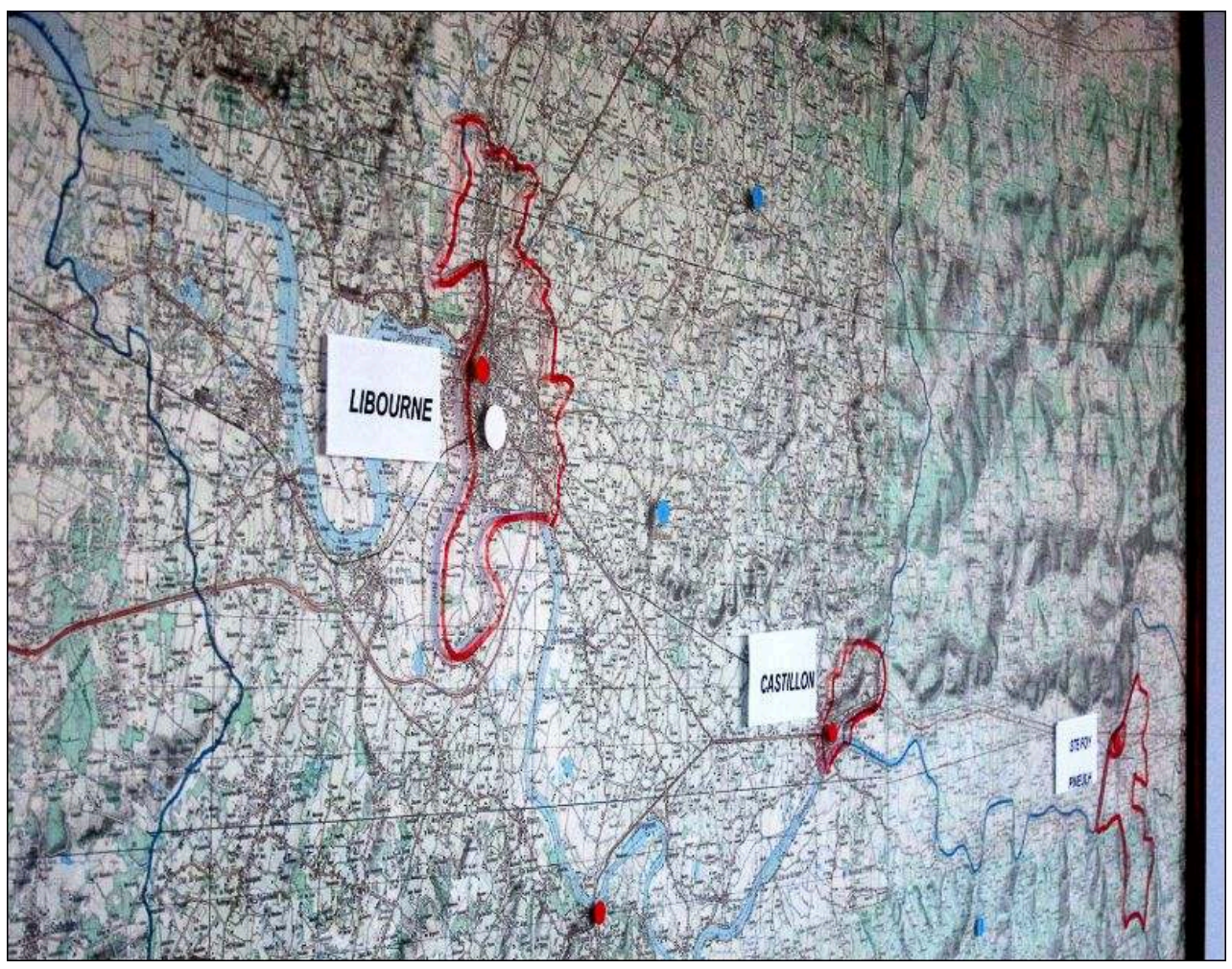

Points rouges et bleus : localisation des brigades. Contours bleus : territoire de la compagnie. Contours rouges : communes bénéficiant du dispositif des Zones de Sécurité Prioritaires.

Auteur: M. Germes, 2013. Réalisation : Carte topographique IGN, annotée par le Commandant de Compagnie dans le bureau duquel elle est affichée.

42 La fonction attribuée à ces représentations affichées dans des bureaux de commandement de la gendarmerie départementale est de donner des points de repères fondamentaux sur l'imbrication spatiale des compétences et sur les contiguïtés spatiales - ce que la gendarmerie a de commun avec les services publics en général. Ici, le «territoire » est un élément essentiel de la définition de la mission de police, il est l'objet même du travail de « terrain »

\section{Cartographie administrative de l'activité : rendre compte des chiffres}

43 Le deuxième type de carte, la cartographie administrative de l'activité, est inséré dans des rapports. Les images, des cartes choroplèthes au milieu d'un flux de textes, de chiffres, de tableaux, mettent en œuvre le même maillage territorial et représentent une information quantitative discrétisée. Lorsque les crimes et délits cartographiés correspondent à la nomenclature de l'État 4001, la base de données de référence pour l'établissement de telles cartographies est Infocentre. Les cartes des vols de métaux ou des vols de fret (non recensés dans l'État 4001) établies par l'OCLDI (Office Central de Lutte contre la Délinquance Itinérante) proposent des cartographies à l'échelle nationale et selon un maillage régional établies avec les données issues de remontées d'information volontaires, ni systématiques ni exhaustives.

44 La fonction de ces cartes est de rendre compte de l'activité de l'institution sur des rythmes assez courts (un an, un mois). Insérées dans des rapports, elles sont destinées à la hiérarchie institutionnelle et au mandataire (préfecture, ministère), qui interprètent 
les «bons » ou «mauvais » chiffres de telle ou telle circonscription dans une logique d'évaluation : en ce sens, la remise de rapports constitue un rituel hiérarchique. Parfois les auteurs ou les destinataires des cartes tentent, pour les premiers, des analyses des phénomènes cartographiés (cf. paragraphe sur les nuages de points), ou considèrent, pour les seconds, qu'elles peuvent aider à la décision.

\section{Synthèse : compétence territoriale et image de l'institution}

La cartographie administrative représente le territoire au sens premier d'espace de compétence sous forme d'un emboîtement hiérarchique de "poupées russes" spatiales, à l'image de l'organisation de l'institution. L'espace est mis en ordre, approprié sans interstice ni fissure et attribué à une unité organisationnelle de la gendarmerie. Le signe graphique du polygone est le moyen de visualiser le maillage territorial. Ce maillage est d'ailleurs construit et redécoupé à partir d'autres maillages étatiques - ce ne sont ni des toponymes, ni des paysages illustrés ou décrits, ni des coordonnées en latitude et longitude, ni des éléments de topographie qui délimitent les territoires de compétence: ce sont des emboîtements de communes, dont le rattachement à des brigades puis à des compagnies est discuté à partir du puzzle du maillage communal et dans le carcan du maillage départemental.

La cartographie administrative repose sur un type bien particulier d'abstraction de l'espace: les polygones matérialisent des espaces-containers ${ }^{10}$ (Wardenga, 2006). L'identité de l'institution (à travers son organisation hiérarchico-spatiale) et l'identité de la fonction de police (et des compétences de surveillance, d'intervention...) sont affirmées par la reproduction de polygones aux contours toujours identiques. Le dessin ainsi répété à l'envi fait correspondre l'« unité territoriale» (brigade, compagnie, groupement) et l'espace, comme si l'une relevait intrinsèquement de l'autre. Le polygone devient une évidence graphique qui conforte le projet territorial. L'affichage en grand de ces cartes en particulier dans les bureaux des commandants d'unité conforte le rôle identitaire de l'image. Cependant, ce rôle reste discret et pragmatique : les cartes ne font l'objet aucune esthétisation, d'aucun encadrement, elles ne sont pas appréciées pour leurs dimensions symboliques, mais mobilisées comme de simples outils de travail énonçant une évidence apparemment sans intérêt majeur.

\section{Les nuages de points de la cartographie d'analyse des "problèmes » : l'adversité}

Dans le vocabulaire de gendarmerie, le terme "analyse " désigne la mission qui consiste à analyser les données policières de la délinquance et de la criminalité, soit dans une démarche de prévention, de planification opérationnelle, soit dans une démarche d'enquête de police judiciaire. La démarche "d'analyse criminelle » repose sur la recherche d'explications, de causalités, de motifs - qui peuvent reposer sur des théories (géo)criminologiques (cf. plus haut), Elle a été massivement introduite dans le travail de police au cours du $20^{\mathrm{e}}$ siècle; elle ne fait pas partie des méthodes traditionnelles de la gendarmerie dont les officiers sont encore essentiellement formés à la "Méthode de Raisonnement Tactique $»^{11}$ militaire, à la portée essentiellement opérationnelle. Cependant, des postes d'analyse criminelle sont créés dans les groupements de gendarmerie, au sein des Brigades Départementales de Renseignement et d'Information Judiciaire (BRDIJ); les mêmes BDRIJ produisent des analyses 
périodiques de la criminalité. Au-delà, des gendarmes, commandants de compagnie ou de brigade s'essaient également à l'analyse, ce qui est probablement lié à une évolution de la culture du métier via des exemples internationaux connus en formation continue ou dans les médias. La cartographie d'analyse désigne les documents qui localisent des événements (crimes, délits, accidents ou événements dans le cadre d'une enquête judiciaire) pour répondre à une question précise. La localisation est souvent évoquée comme un élément explicatif et la carte comme un élément probant. En voici trois exemples.

\section{Accidentologie routière}

La cartographie de l'accidentologie routière représente les accidents par des points sur un fond de carte routier simplifié du département par exemple. Les gendarmes des Escadrons Départementaux de Sécurité Routière (ESDR) font varier les figurés selon le type de véhicule impliqué, gravité de l'accident (matériel, corporel, mortel) et en fonction des causes attribuées à l'accident (vitesse, alcool, priorité...). Ces documents, réalisés en $\mathrm{CAO}$ et intégrés à un rapport au milieu de chiffres, tableaux et textes, sont actualisés selon le rythme mensuel des orientations stratégiques des politiques de sécurité routière décidées par le commandant de groupement. La cartographie est un élément parmi d'autres tableaux statistiques dont le rôle dans la prise de décision sur la localisation des contrôles dépend de l'interprétation de la hiérarchie.

\section{Cartographie des crimes et délits}

Sur le même principe mais dans un autre domaine, la cartographie des crimes et délits recensés (cf. illustration 4) est établie tantôt par ou pour des commandants de brigade et de compagnie, ou bien réalisées par des BDRIJ pour les commandants de groupement. La plupart du temps ce sont les cambriolages et les vols, évènements suffisamment fréquents pour être cartographiés, attirant suffisamment l'attention des autorités administratives et des élus - mais aussi évènements qui sont associés à une explication par l'espace et donc à une représentation cartographique. Les auteurs sont censés avoir une stratégie spatiale dont le décodage peut permettre l'interpellation ou la prévention. Ces cartes pourraient en principe être établies automatiquement (en suivant la nomenclature des infractions de l'État 4001) à partir de la base de données des statistiques de l'activité de la police Infocentre, si le géocodage des évènements dans Infocentre était exhaustif. Ainsi, les données cartographiées sont le plus souvent constituées manuellement, dans des tableurs via le suivi des procès-verbaux au jour le jour portant sur des thèmes précis. Les supports numériques interactifs sont privilégiés (comme Via Michelin ou Google Maps; plus rarement des cartes topographiques digitalisées), la localisation des événements par points est faite également manuellement. Les figurés changent parfois en fonction des variables disponibles, limitées en général au moment de l'infraction et au type de lieu où elle a été commise (résidence, entreprise, établissement public). 


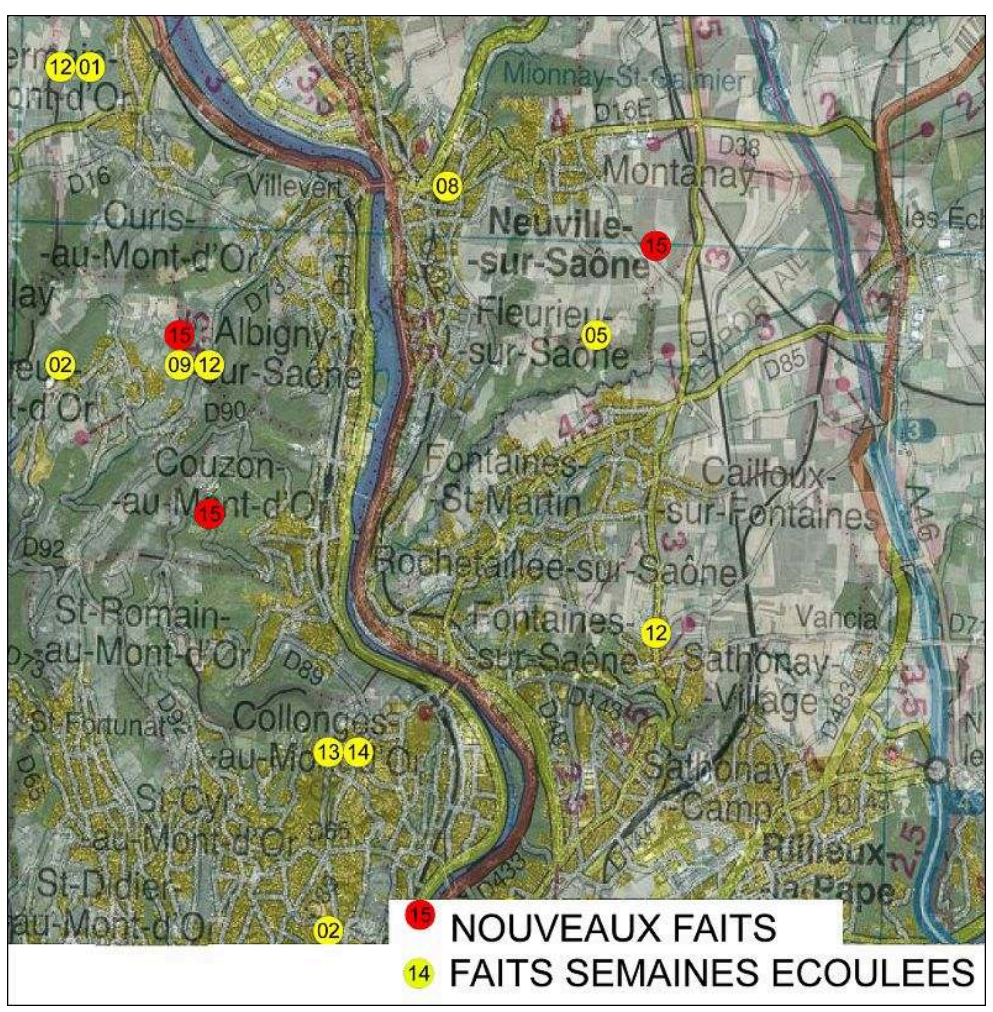

Le fond de carte Michelin est constitué d'une superposition par transparence d'une vue aérienne et d'une carte routière. Les points sont ajoutés et numérotés manuellement.

Source : copie d'écran recoupée d'un document image, BDRIJ.

La carte ainsi produite constitue une image de travail, que l'on transmet en fonction des besoins, ou que l'on garde pour soi lorsqu'elle n'est pas concluante. La finalité est d'établir un document qui contribue à l'analyse d'un type de crime ou de délit particulier, de façon à déterminer les orientations stratégiques de la lutte contre un type de phénomène, en espérant identifier des modèles spatiaux permettant l'anticipation et la surveillance. Il s'agit en fait d'un modèle hybride d'informatisation de la classique "pin map ", et non d'une crime map au sens où l'entendent les experts.

\section{Cartographie d'analyse pour enquête judiciaire}

51 Le troisième exemple est la cartographie d'analyse criminelle dans le cadre d'une enquête judiciaire. Les données issues des enquêtes et des réquisitions (telles les factures détaillées des connexions téléphoniques) sont manipulées par un analyste criminel, qui choisit parfois de les représenter sous forme cartographique. Les cartes représentent la correspondance spatiale entre un événement (dans un lieu et à un moment donné) et les connexions téléphoniques de numéros particuliers. Sur un fond de carte, routier ou topographique, des points événements sont reliés par des flèches directionnelles, reconstituant un trajet. Différentes séries d'évènements sont superposées et mises en correspondance. Ces documents présentent le résultat de l'analyse de données formelles (travaillées sous forme de tableurs ou de bases de données) et mettent en forme de manière concise des informations destinées aux enquêteurs et aux juges. Elles font partie d'un dossier conséquent, constitué de multiples procès-verbaux et de nombreuses pages de textes, et constituent un 
« résumé », une " simplification », une " explication » plus simple que la démonstration textuelle et argumentative qui les accompagne.

Illustration 5 - Itinéraire de deux téléphones : extrait d'une carte représentant l'activation d'antennes de relais téléphoniques par deux téléphones portables dans le contexte d'une enquête de police judiciaire

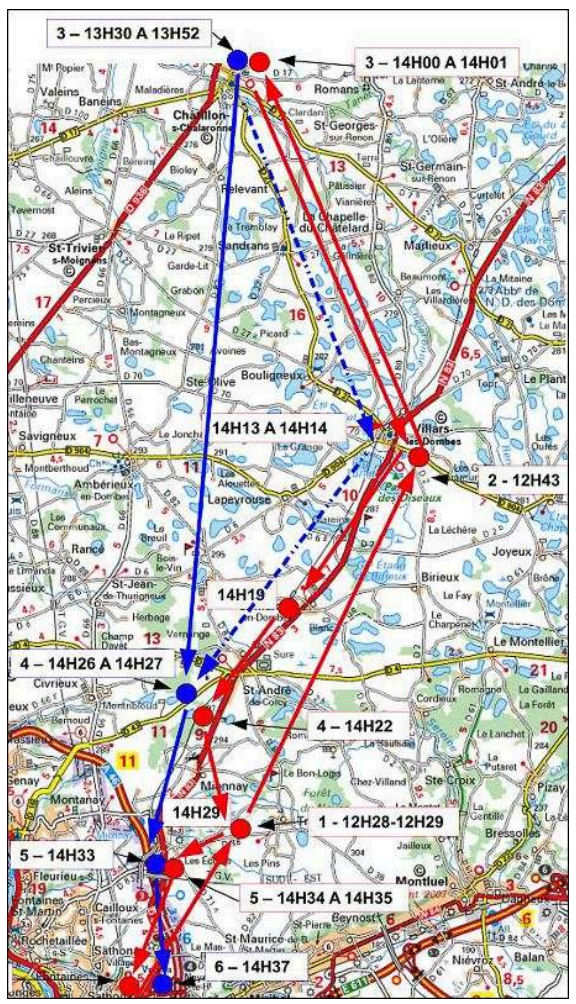

La carte a été un élément du témoignage de l'enquêteur devant le tribunal, en pièce annexe au procèsverbal.

\section{Synthèse : la mobilité comme problème, la visualisation comme outil}

La cartographie d'analyse répond à un projet de connaissance : il s'agit de localiser pour interpréter et expliquer, de deux manières. Les cartographies d'analyse de police judiciaire doivent contribuer à résoudre des enquêtes dans un contexte judiciaire, les cartographies d'analyse de la criminalité et de l'accidentologie servent à engager et à diriger des moyens de coercition, de surveillance, de prévention. Le nuage de points est l'opposé du polygone : alors que ce dernier évoque contrôle, présence et responsabilité, le nuage de points désordonnés et éparpillés évoque la spontanéité, le désordre, la déviance qui a échappé au contrôle. Le nuage de points confronte le lecteur à la complexité de l'espace social, il mobilise son savoir professionnel pour donner du sens, pour tenter d'expliquer en fonction de correspondances spatiales avec d'autres éléments - les autoroutes, les quartiers chics et les quartiers sensibles, les jeunes désœuvrés... Ce savoir professionnel constitue le fond de carte invisible que restitue le récit autour de la carte, et qui est transmis par les interlocuteurs à l'oral («ce qu'il faut savoir... »). Le grand espoir de l'analyste est que les actes isolés (les points) aient un sens, une logique, une cohérence. La carte par points n'est qu'un commencement qui appelle une mise en ordre graphique : d'où le dessin de lignes et de cercles qui sont 
autant d'hypothèses d'un ordre sous-jacent et de tentatives de reconstitution d'un principe organisateur.

La cartographie d'analyse repose sur un autre type particulier d'abstraction de l'espace: la manipulation des nuages de points exprime la recherche d'une causalité spatiale, d'une forme de déterminisme spatial ${ }^{12}$. Les nuages de points expriment ainsi l'adversité : ils devraient révéler les structures spatio-temporelles de la délinquance et permettre de comprendre les logiques spatiales d'un problème. La mobilité est considérée comme une ressource pour les criminels et délinquants : l'espace livre sinon une variable explicative, du moins un schéma (pattern). Les nuages de points sont des traces laissées dans l'espace d'une territorialité déviante, mobile et insaisissable. La carte de la criminalité présuppose que cette répartition a un sens et appelle une interprétation cohérente qui permette de désigner les espaces de la prochaine intervention ou surveillance. Les points ne sont pas seulement des artefacts, mais des indices, porteurs de sens, à déchiffrer. Ces cartes construisent une territorialité antagonique et représentent la mission policière.

\section{Les champs de force de la cartographie opérationnelle : la maîtrise}

Dans le vocabulaire de gendarmerie, le terme " opérationnel » renvoie à l'intervention lors d'un événement précis avec une mission particulière : si la situation est mal connue et peu maîtrisée, les objectifs et "conduites à tenir » sont clairs. La cartographie opérationnelle désigne le recours à des cartes de localisation, leur actualisation et leur adaptation dans le but d'intervenir et de coordonner la présence effective sur le terrain de l'intervention - la fonction très ancienne de la carte d'État-Major est étroitement liée à la conception hiérarchique d'une chaîne de commandement, comme outil indispensable au contrôle institutionnel de l'espace, au-delà des perspectives limitées de chaque acteur isolé. Les acteurs ont rarement recours aux cartes topographiques mais ils privilégient les images aériennes (par opposition aux plans) car ils leur donnent un aperçu du « paysage » et s'approchent le plus d'une reconstitution virtuelle de la réalité.

\section{Localiser}

Même si les gendarmes accordent beaucoup d'importance à la connaissance du territoire de la brigade (pour les unités territoriales) pour se diriger, les cartographies de localisation géographique sur support papier (cartes routières, plans de ville, voire plans à partir de sites internet) permettent d'avoir sur le terrain une vue surplombante de la situation. Par ailleurs, les cartographies des équipements embarqués (GPS, ordinateurs reliés au réseau) permettent de relier au cours d'un déplacement l'unité isolée au centre opérationnel.

\section{Intervenir}

Le deuxième cas est un perfectionnement du premier:les cartographies d'interventions servent à coordonner l'intervention et à transmettre des ordres. Par exemple, les opérations de maintien de l'ordre imposent le recours à des cartographies lorsqu'une unité spécialisée se déplace pour un évènement particulier, souvent préparé à l'avance. La cartographie fait partie de l'ordre de mission, elle illustre les instructions 
sur les « conduites à tenir ", donne des éléments d'explication du contexte et permet aux acteurs de terrain de se positionner par rapport à leurs collègues, aux personnalités à protéger, aux foules à encadrer, aux dangers à identifier, dans un espace qui ne leur est pas forcément familier ou dans un dispositif qu'ils n'ont pas encore expérimenté. Les supports - plans de ville ou vues aériennes - sont complétés par un dessin manuel et une légende. Le commandement local s'adresse ainsi directement aux gendarmes dans le cas d'une reconstitution pour une enquête de police judiciaire, de l'organisation d'une interpellation, ou d'une mission de maintien de l'ordre, ou de plans saisonniers d'intervention.

Illustration 6 - Reconstitution judiciaire

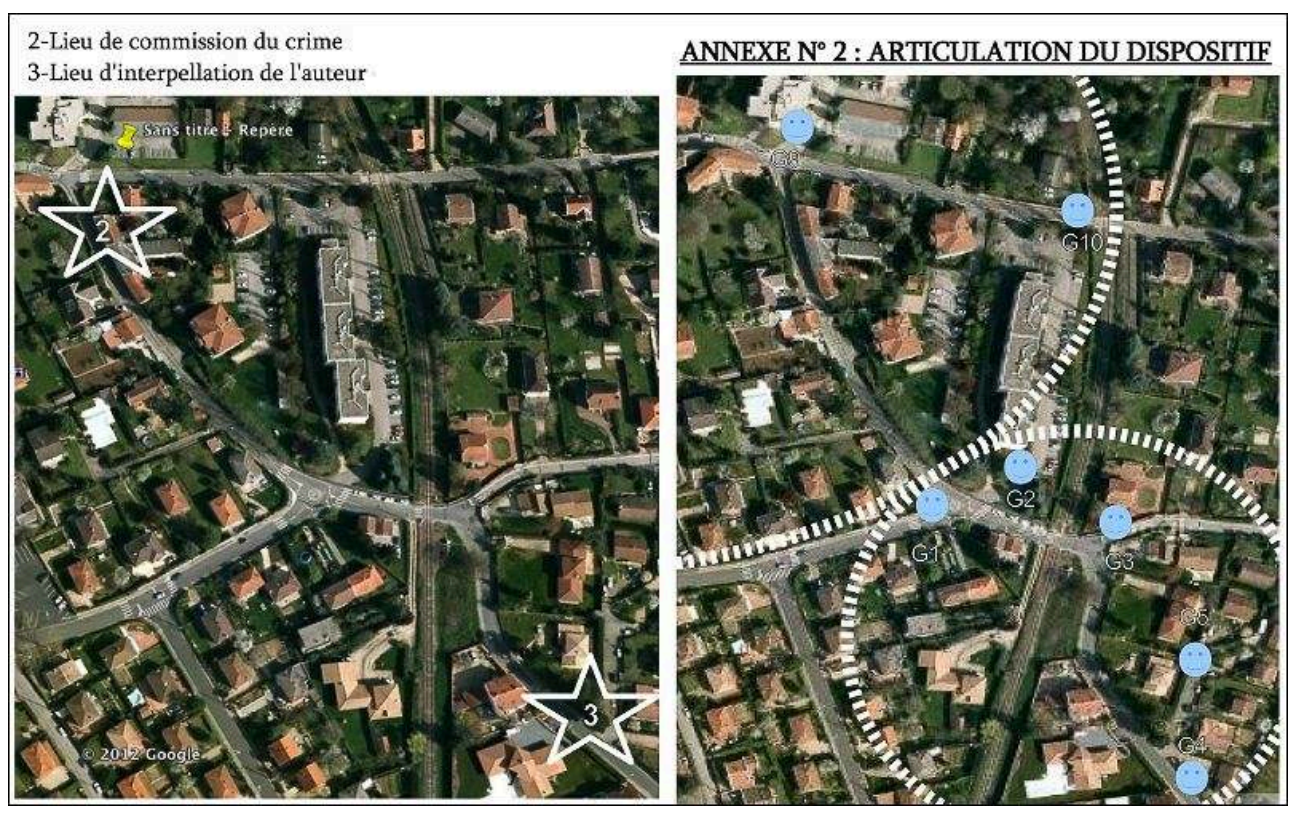

Extraits de deux documents accompagnant un ordre de mission dans le cadre d'une reconstitution judiciaire.

Réalisation : Brigade Recherche.

\section{Cartographie tactique}

57 Le troisième exemple est la cartographie tactique des forces en présence. Les cartes d'État-Major ou cartes topographiques IGN en furent longtemps les seuls outils (cf. illustration 1), aujourd'hui elles sont complétées par la cartographie opérationnelle de la base de données en ligne BDSP (cf. illustration 2), qui géolocalise en temps réel tous les véhicules de gendarmerie dotés d'une balise et tous les appels au 17 entrants. Ces cartographies correspondent à la fonction de l'État-Major, remplie aujourd'hui au niveau départemental par les CORG (Centre d'Opérations et de Renseignement de la Gendarmerie), où une salle d'appel (cf. illustration 1) et une salle de cellule de crise permettent à la fois la coordination des interventions au jour le jour et l'engagement de renforts en situation de crise. Les CORG ont une vision d'ensemble du terrain et de l'ensemble des interventions en cours, dont les progrès sont signalés depuis les véhicules. 


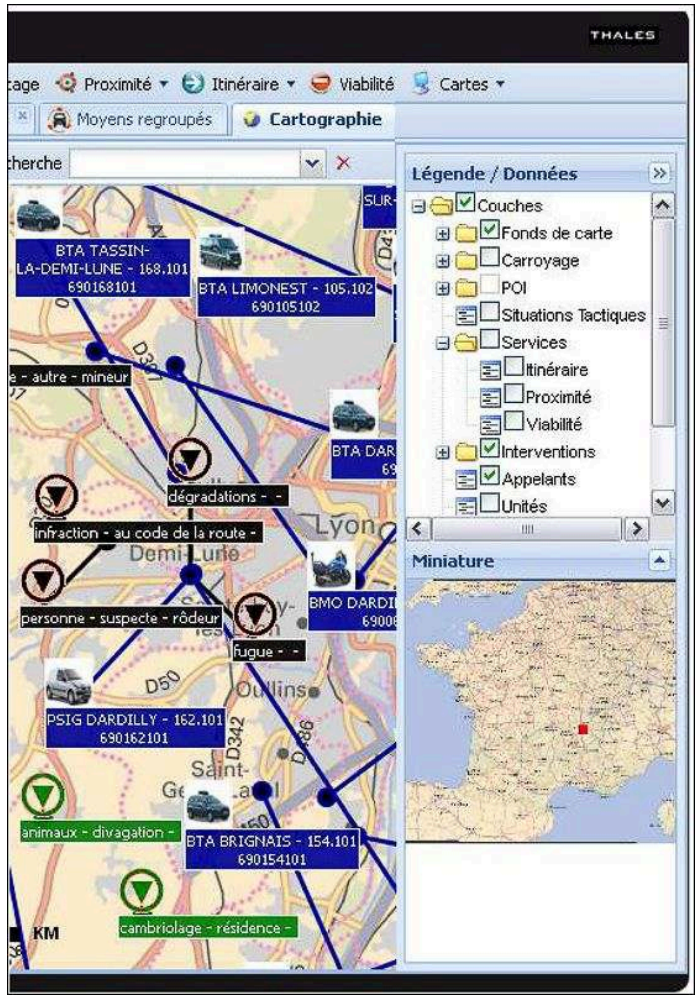

Copie d'écran recoupée du module cartographique de la base de données BDSP, représentant l'ensemble des événements en cours et conçue pour coordonner l'intervention en temps réel des unités de gendarmerie. Les traits relient les unités (représentées par une image et un nom) à leur géolocalisation (point).

\section{Synthèse : maîtriser la configuration de l'interaction via la représentation}

La cartographie opérationnelle remplit une fonction pragmatique en aidant à diriger l'action dans un espace où les évènements évoluent en permanence : il s'agit d'orienter et de décider de son propre déplacement, le déplacement d'autres unités ou de structurer la tactique d'une intervention dans une situation incertaine. En accompagnement des ordres (ordres de mission ou ordres par radio), elle entretient un lien entre les gendarmes sur le terrain (qui jouissent d'un important pouvoir discrétionnaire) et l'institution, les autres unités, la hiérarchie. Elle est un instrument de commandement et d'information - quel que soit l'événement en cours : accident de la route, catastrophe naturelle ou trouble à l'ordre public - et ainsi étroitement liée aux véhicules et à la radio. La temporalité de l'utilité de la cartographie opérationnelle consiste à appréhender le présent pour décider d'actions quasiment immédiates - alors que la cartographie administrative et la cartographie d'analyse sont structurées par le passé dans les cadres temporels arbitraires de la comptabilité statistique. Cette cartographie répond à la fois au besoin de structuration d'une action collective complexe sur un espace topographiquement stable mais dont les configurations sont changeantes et au besoin d'une connaissance du territoire.

C'est sur un troisième type d'abstraction de l'espace que repose la cartographie opérationnelle : sur des champs de force provisoires. Ils structurent les interactions en départageant différents groupes d'acteurs susceptibles d'intervenir, et les objets de 
l'action (protection ou surveillance). Sans être dessinés sur les documents et images, qui représentent des points, des zones, des lignes de parcours, ils sont signifiés par l'usage de couleurs différentes signalant l'appartenance (d'un objet, d'une position, d'un parcours) à un groupe ou un autre. Ils expriment l'idée, plus relationnelle, d'une topographie des relations ${ }^{13}$. Ils sont provisoires et évoluent en fonction de la reconfiguration des interactions mobiles d'une multitude d'acteurs, conduisant à l'adaptation des décisions. Ils rendent compte de l'absence d'un contrôle complet effectif. Au contraire, il s'agit de tenter la supervision et d'organiser la contrainte, de maintenir d'un équilibre des forces et des positions qui représentent un "ordre » acceptable.

\section{Conclusion : l'abstraction cartographique au service du projet territorial}

Les résultats du terrain pourraient certes être décevants par rapport à l'attente de cartographies policières aussi perfectionnées que le crime mapping. L'insatisfaction de nombreux agents qui répètent au cours de l'enquête ne pas avoir les moyens de réaliser les cartes qu'ils souhaitent en serait confortée. Du point de vue des recherches sur la police focalisées sur les polices anglophones, la gendarmerie nationale française présente certes des spécificités en termes de culture professionnelle qui pourraient paraître dépaysantes. Le but de cette recherche est justement de procéder à un décentrement du regard vers un terrain mal connu, la gendarmerie française et à un déplacement de l'objet, ouvert à l'ensemble des pratiques cartographiques. En combinant les apports des recherches en sciences sociales sur la police et la cartographie, tant dans le monde francophone, anglophone et germanophone, j'ai pu montrer que les pratiques cartographiques font d'abord partie d'une culture visuelle ordinaire au sein de la gendarmerie et que leur mobilisation résulte d'une pratique plus vernaculaire qu'experte ou savante. Il s'avère alors nécessaire d'adopter une approche de cartographie critique et de méthodes d'observation qui montrent quelles sont les pratiques quotidiennes du territoire de l'État. Si la cartographie policière fait certes partie d'un projet territorial (de contrôle et de souveraineté), la mise en œuvre de ce dernier doit composer avec de nombreuses contingences organisationnelles, institutionnelles et culturelles. Il s'agit alors de mettre l'accent sur les interprétations, significations et rôles attribuées aux cartes et sur les croyances qui poussent les agents à trouver des arrangements (tels que la collecte manuelle de données et les montages logiciels) pour produire, malgré les difficultés matérielles, des cartographies. Cependant, dans la majorité des cas, la lecture des cartes consiste à mobiliser l'image pour confirmer une représentation existante, mise visuellement en évidence. La recherche a montré que la cartographie remplit trois fonctions complémentaires dans le travail de police: un rôle administratif, d'analyse et opérationnel. Ces fonctions correspondent à trois projets territoriaux complémentaires: la construction de l'identité de l'institution dans l'espace; la construction de l'adversité dans l'espace; l'enjeu de la maîtrise de l'espace. 
Tableau 1 - Trois abstractions de l'espace pour les trois facettes d'un projet territorial

\begin{tabular}{|l|l|l|l|l|}
\hline $\begin{array}{l}\text { Type de cartographie } \\
\text { policière }\end{array}$ & $\begin{array}{l}\text { Cartographie } \\
\text { administrative }\end{array}$ & Cartographie d'analyse & $\begin{array}{l}\text { Cartographie } \\
\text { opérationnelle }\end{array}$ \\
\hline Exemples & $\begin{array}{l}\text { Territoire de } \\
\text { compétence; comptes- } \\
\text { rendus }\end{array}$ & $\begin{array}{l}\text { Accidentologie routière; ; } \\
\text { judiciare }\end{array}$ & $\begin{array}{l}\text { Localisation; } \\
\text { intervention; } \\
\text { tactique }\end{array}$ \\
\hline Abstraction & graphique & Polygone & Nuage de points & Champ de forces \\
\hline conceptuelle & Espace container & Déterminisme spatial & $\begin{array}{l}\text { Topographie des } \\
\text { relations }\end{array}$ \\
\hline Projet territorial & $\begin{array}{l}\text { Identité } \\
\text { construction } \\
\text { l'institution }\end{array}$ & $\begin{array}{l}\text { Construction } \\
\text { l'adversité }\end{array}$ & deîtrise de l'espace \\
\hline
\end{tabular}

61 Ces trois projets sont identifiés grâce à la notion d'abstraction cartographique selon B. Belina qui désigne trois phénomènes souvent imbriqués (Belina, 2009, p. 195-196). Premièrement et de manière très générale, l'abstraction du réel inhérente et nécessaire à la représentation cartographique (sélection, réduction, classification, généralisation, etc.). Dans un deuxième temps et très fréquemment, l'abstraction par réification qui tend à effacer tant la construction de la carte que la construction sociale des phénomènes cartographiés. Dans un troisième temps et de manière assez courante, selon la manière dont le document est mis en œuvre, la "fausse abstraction » que constitue le fétichisme spatial, c'est-à-dire la réduction du social au spatial. Les représentations sont alors marquées par des biais idéologiques qui en font des contrevérités, et selon les pratiques des acteurs qui les utilisent et leur pouvoir, elles sont des abstractions «devenues vraies par la pratique $»^{14}$ contribuant à la mise en œuvre concrète de l'hégémonie qu'elles portent.

62 J'ai décomposé l'analyse des abstractions cartographiques en deux temps (cf. tableau cidessus) : premièrement, l'analyse des formes graphiques (polygones, nuages de points, champs de force), deuxièmement, l'analyse des concepts spatiaux sous-jacents (container, déterminisme, topographie), et ainsi mettre en valeur trois projets territoriaux combinés. Chacune de ces abstractions est caractérisée par la naturalisation du phénomène cartographié (la construction politique et institutionnelle des circonscriptions, des objets de l'analyse et des acteurs en situation opérationnelle sont invisibilisés par la carte). L'identité, l'adversité et la maîtrise apparaissent de plus comme trois de ces «fausses » abstractions (qui reposent entre autres sur de « fausses » conceptions de l'espace, en termes de container, de déterminant, ou de topographie des relations) dont la mise en œuvre cartographique est utile aux pratiques policières concrète dans le monde réel.

63 Au-delà d'une conception de la construction du territoire comme une simple mise en place des instruments de la souveraineté étatique dans l'espace, il me semble pertinent d'admettre que le projet étatique de puissance, d'appropriation voire de coercition est soutenu d'abord par la routine et la dimension vernaculaire des pratiques quotidiennes de ses agents contraints à s'arranger avec le réel. L'expression « projet territorial » met 
l'accent sur le fait que si l'appropriation de l'espace apparaît comme une nécessité, elle est par définition non-réalisée et fondamentalement incomplète, car confrontée à l'adversité, à des contestations politiques, à des difficultés organisationnelles: le territoire (étatique ou policier) est un exercice quotidien.

\section{BIBLIOGRAPHIE}

Aldhuy J., 2008. Au-delà du territoire, la territorialité? Géodoc, , 55, p. 35-42.

Antonsich M., 2011. Rethinking territory. Progress in Human Geography, 35, 3, p. 422-425. doi: 10.1177/0309132510385619.

Bautes N., Marie Dit Chirot C., 2012. Pour une géographie sociale de l'action. Carnets de géographes, 4, http://www.carnetsdegeographes.org/carnets_recherches/rech_04_01_Bautes.php. Consulté le 22.01.2014

Beaude B., 2009. Crime Mapping, ou le réductionnisme bien intentionné. EspacesTemps.net, http:// www.espacestemps.net/articles/crime-mapping-ou-le-reductionnisme-bien-intentionne/. Consulté le 20.03.2014.

Behr R., 2006. Polizeikultur. Routinen, Rituale, Reflexionen. Bausteine zu einer Theorie der Praxis der Polizei. VS Verlag für Sozialwissenschaften, Wiesbaden, $211 \mathrm{p}$.

Belina B., 2000. "Kriminalität" und "Raum". Zur Kritik der Kriminalgeographie und zur Produktion des Raums. Kriminalistisches Journal, 32, 2, p. 129-147.

Belina B., 2003. Evicting the Undesirables. The Idealism of Public Space and the Materialism of the Bourgeois State. BelGeo, 1, p. 47-62.

Belina B., 2006. Raum, Überwachung, Kontrolle: vom staatlichen Zugriff auf städtische Bevölkerung. Westfälisches Dampfboot, Münster, 321 p.

Belina B., 2008. Kritische Geographie: Bildet Banden! Einleitung zum Themenheft. ACME: An International E-Journal for Critical Geographies, 7, 3, p. 335-349, http://www.acme-journal.org/vol7/ Belina.pdf . Consulté le 20.03.2014.

Belina B., 2009. Kriminalitätskartierung - Produkt oder Mittel neoliberalen Regierens, oder: Wenn falsche Abstraktionen durch die Macht der Karte praktisch wahr gemacht werden. Geographische Zeitschrift, 4, p. 192-212.

Belina B., 2013. Raum : Zu den Grundlagen eines historisch-geographischen Materialismus. Westfälisches Dampfboot, Münster, 173 p.

Belina B., Miggelbrink J., 2010. Hier so, dort anders. In Belina B., Miggelbrink J. (coord.), Hier so, dort anders - Raumbezogene Vergleiche in dewr Wissenschaft und anderswo. Verlag Westfälisches Dampfboot, Münster, p. 7-39.

Besson J.-L., 2005. Les cartes du crime. Presses Universitaires de France, Paris, 288 p.

Brantingham P., Andresen M., Kinney, B. (coord.), 2010. Classics in environmental criminology. SFU Publications, Burnaby, B.C, 546 p. 
Brantingham P., Brantingham P. (coord.), 1991 [1981]. Environmental Criminology. Waveland Press, Prospects Heigh, 262 p.

Brodeur J.-P., 2001. Le travail d'Egon Bittner : une introduction à la sociologie de la force institutionnalisée. Déviance et Société, 25, 3, p. 307. doi:10.3917/ds.253.0307.

Brodeur J.-P., 2003. Les visages de la police. Pratiques et Perceptions. Les Presses de l'Université de Montréal, Montréal, 396 p.

Brodeur J.-P., Monjardet D. (coord.), 2003. Connaître la police: Grands textes de la recherche anglosaxonne. Les Cahiers de la Sécurité intérieure, Institut des Hautes Études de la Sécurité intérieure, Paris, $453 \mathrm{p}$.

Camilleri G., Lazerges C., 1992. Atlas de la criminalité en France. Mappemonde, 2, p. 6-9.

Chainey S., Tompson L. (coord.), 2008. Crime mapping case studies : Practice and research. John Wiley \& Sons, Chichester, England, Hoboken, NJ, 162 p.

Chan J., 2004a. Police and new technologies. In Newburn T. (coord.), Handbook of Policing. Willan Publishing, Cullompton, p. 655-679.

Chan J., 2004b. Using Pierre Bourdieu's Framework for unterstanding Police Culture. Droit et Société, 1, p. 327-346. http://www.cairn.info/revue-droit-et-societe-2004-1-page-327.htm. Consulté le 01.08.2011.

Collignon B., 2005. Que sait-on des savoirs géographiques vernaculaires? Bulletin de l'Association de Géographes Francais, 3, p. 321-331.

Crampton J.W., 2009. Cartography: maps 2.0. Progress in Human Geography, 33, 1, p. 91-100. doi: 10.1177/0309132508094074.

Cunty C., Fussy F., Perez, P., 2007. Géocriminologie, quand la cartographie permet aux géographes d'investir la criminologie. Cybergeo: Revue européenne de géographie, 378, http:// www.cybergeo.eu/index7058.html. Consulté le 20.03.2014.

Debarbieux B., 1999. Le territoire : Histoires en deux langues. In Chivallon C., Ragouet P., Samers M. (coord.), Discours scientifiques et contextes culturels. Géographies britanniques et françaises à l'épreuve postmoderne. Maison des sciences de l'homme d'Aquitaine, Talence, France, p. 33-34.

Del Casino V.D., Hanna S.P., 2005. Beyond The 'Binaries': A Methodological Intervention for Interrogating Maps as Representational Practices. ACME: An International E-Journal for Critical Geographies, 4, 1, p. 34-56. http://www.acme-journal.org/vol4/VDCSPH.pdf . Consulté le 12.03.2013.

Di Méo G., 1999. Géographies tranquilles du quotidien : Une analyse de la contribution des sciences sociales et de la géographie à l'étude des pratiques spatiales. Cahiers de géographie du Québec, 43, 118, p. 75-93.

Didier E., 2011a. « Compstat » à Paris : Initiative et mise en responsabilité policière. Champ pénal / Penal Field, VIII, doi:10.4000/champpenal.7971.

Didier, E., 2011b. L'État néolibéral ment-il ? « Chanstique » et statistiques de police. Terrain, 57, p. 66-81. doi:10.4000/terrain.14338.

Eick V., Sambale J., Töpfer, E. (coord.), 2007. Kontrollierte Urbanität : Zur Neoliberalisierung städtischer Sicherheitspolitik. transcript, Bielefeld, 398 p.

Elden S., 2010. Thinking Territory Historically. Geopolitics, 15, 4, p. 757-761. doi: 10.1080/14650041003717517. 
Evans D.J., Fyfe, N.R., Herbert, D.T. (coord.), 1992. Crime, Policing and Place. Essays in environmental criminology. Routledge, London, New York, $332 \mathrm{p}$.

Ferret J., Mouhanna C. (coord.), 2005. Peur sur les villes. Presses Universitaires de France, Paris, $230 \mathrm{p}$.

Field K., 2011. Reflections on a Criminal Crime Map : Editorial. The Cartographic Journal, 48, 1, p. 1-3. doi:10.1179/174327711X13052865724329.

Fyfe N.R., 1991. The police, space and society: the geography of policing. Progress in Human Geography, 15, 3, p. 249-267. http://phg.sagepub.com

Garland D., 2001. The culture of control: Crime and social order in contemporary society. University of Chicago Press, Chicago, xiii, 307.

Germes M., Glasze G., Weber, F., 2011. « Neue Kulturgeographie » - Débats et perspectives au sein de la nouvelle géographie culturelle germanophone. Cybergeo: Revue européenne de géographie, doi: 10.4000/cybergeo.24727.

Glasze G., Pütz R., Rolfes, M. (coord.), 2005. Diskurs - Stadt - Kriminalität. Städtische (Un-)Sicherheiten aus der Perspektive von Stadtforschung und Kritischer Kriminalgeographie. transcript, Bielefeld,326 p.

Groff E.R., La Vigne N.G., 2002. Forecasting the future of predictive crime mapping. Crime Prevention Studies, 13, consulté le 26.11.2012.

Guerry A.M., 1833. Essai sur la statistique morale de la France. Crochard, Paris,115 p.

Herbert S., 1996. Policing space: Territoriality and the Los Angeles Police Department. University of Minnesota Press, Minneapolis,194 p.

Herbert S., 1997. Territoriality and the Police. The Professional Geographer, 49, 1, p. 86-94. doi: 10.1111/0033-0124.00059.

Herbert S., 1999. The end of the territorially-sovereign state? The case of crime control in the United States. Political Geography, 18, p. 149-172. doi:10.1016/S0962-6298(98)00080-8.

Herbert S., 2003. Coercion, Territoriality, Legitimacy: The Police and the Modern State. In Low, M. (coord.), Handbook of political geography. United States, Sage Publications, p. 169-181.

Herb G.H., Häkli J., Corson M.W., Mellow N., Cobarrubias S. et Casas-Cortes, M., 2009. Mapping is critical! Political Geography, 28, 6, p. 332-342. doi:10.1016/j.polgeo.2009.09.005.

Howard R.G., 2008. The Vernacular Web of Participatory Media. Critical Studies in Media Communication, 25, 5, p. 490-513. doi:10.1080/15295030802468065.

Jobard, F., 2005. Le nouveau mandat policier : Faire la police dans les zones dites de «non-droit ». Criminologie, 38, 2, p. 103-121. http://id.erudit.org/iderudit/012664ar.

Joliveau T., Noucher M., Roche, S., 2013. La cartographie 2.0, vers une approche critique d'un nouveau régime cartographique. L'Information géographique, 77, 4, p. 29-46. doi:10.3917/lig. 774.0029 .

Lascoumes P., Le Galès P., 2004. L'action publique saisie par les instruments. In Lascoumes P., Le Galès P. (coord.), Gouverner par les instruments. Presses de Sciences Po, Paris, p. 11-44.

Le Goff T., 2013. Dans les « coulisses » du métier d'opérateur de vidéosurveillance. Criminologie, 46, 2, p. 91. doi:10.7202/1020988ar. 
Manning P.K., 2001. Technology's Ways : Information Technology, Crime Analysis and the Rationalizing of Policing. Criminology and Criminal Justice, 1, 1, p. 83-103. doi: $10.1177 / 1466802501001001005$.

Mazerolle L.G., Bellucci C., Gajewski, F., 1997. Crime Mapping in police Departments: The Challenges of Building a Mapping System. Crime Prevention Studies, 8, p. 131-155. Consulté le 26.11.2012.

Mensching A., 2008. Gelebte Hierarchien. Mikropolitische Arrangements und organisationskulturelle Praktiken am Beispiel der Polizei. VS Verlag für Sozialwissenschaften, Wiesbaden, 355 p..

Monjardet D., 1994. La culture professionnelle des policiers. Revue française de Sociologie, 35, 3. Monjard et D., 1996. Ce que fait la police : Sociologie de la force publique. La Découverte, Paris, 316 p. Morange, M., Calbérac, Y., 2012. Géographies critiques "à la française" ? Carnets de géographes, 4 , http://www.carnetsdegeographes.org/PDF/debat_04_01_Morange_Calberac.pdf. Consulté le 18.11.2013.

Moreau Saint-Martin L. de, Garnier A., 2001. Sur la normalisation de la prévention de l'insécurité par l'urbanisme. Les Cahiers de la sécurité intérieure, 43, 1, p. 9-17.

Mucchielli L. (coord.), 2008a. La frénésie sécuritaire. Retour à l'ordre et nouveau contrôle social. La Découverte, Paris, $140 \mathrm{p}$.

Mucchielli L., 2008b. Le « nouveau management de la sécurité » à l'épreuve : délinquance et activité policière sous le ministère Sarkozy (2002-2007). Champ pénal/Penal Field, , Vol. V, doi: 10.4000/champpenal.3663.

Mucchielli L., Robert P. (coord.), 2002. Crime et sécurité : L'état des savoirs. Editions La Découverte, Paris, $438 \mathrm{p}$.

Neocleous M., 2003. Off the Map: On Violence and Cartography. European Journal of Social Theory, 6 , 4, p. 409-425. doi:10.1177/13684310030064003.

Norris C., Armstrong G., 1999. The maximum surveillance society : The rise of CCTV. Berg, Oxford, New York, 248 p.

Painter J., 2010. Rethinking Territory. Antipode, 42, 5, p. 1090-1118. doi:10.1111/j. 1467-8330.2010.00795.x.

Peet R., 1975. The Geography Of Crime: A Political Critique*. The Professional Geographer, 27, 3, p. 277-280. doi:10.1111/j.0033-0124.1975.00277.x.

Perkins C., 2008. Cultures of Map Use. Cartographic Journal, 45, 2, p. 150-158. doi: 10.1179/174327708X305076.

Pickles J., 2004. A history of spaces: Cartographic reason, mapping, and the geo-coded world. Routledge, London, New York, $233 \mathrm{p}$.

Purenne A., Wuilleumier A., 2011. L'introduction des technologies de surveillance dans le travail policier. Facteur de changement ou de réassurance? Droit et Cultures, 61, http:// droitcultures.revues.org/2458. Consulté le 21.11.2013.

Ratcliffe J.H., 2002. Crime Mapping and the Training Needs of Law Enforcement. European Journal on Criminal Policy and Research, 10, 1, p. 65-83. doi:10.1023/B:CRIM.0000037550.40559.1c.

Ripoll F., Veschambre V., 2005. L'appropriation de l'espace comme problématique. Norois, 195, 2, p. 7-15. http://norois.revues.org/477. Consulté le 31.11.2012. 
Rossmo K., Rombouts S., 2008. Geographic profiling. In Wortley R., Mazerolle L.G. (coord.), Environmental criminology and crime analysis. Willan Pub., Cullompton, Devon, UK, p. 136-149.

Wallace A., 2009. Mapping City Crime and the New Aesthetic of Danger. Journal of Visual Culture, 8, 5, p. 5-24. doi:10.1177/1470412908100900.

Wardenga, U., 2006. Raum- und Kulturbegriffe in der Geographie. In Dickel M., Kanwischer D. (coord.), TatOrte. Neue Raumkonzepte didaktisch inszeniert. LIT Verlag, Berlin, p. 21-47.

Weisburd D., Bernasco W., Bruinsma G.J. (coord.), 2009. Putting Crime in its Place : Units of Analysis in Geographic Criminology. Springer New York, New York, NY, 245 p.

Yarwood R., 2007. The geographies of policing. Progress in Human Geography, 31, 4, p. 447-465. doi: 10.1177/0309132507079500.

\section{NOTES}

1. Le terme générique "police» désigne une grande diversité d'institutions publiques qui remplissent des missions de surveillance, d'intervention et d'enquête à propos d'actions illégales. Le problème de la définition de la police a été soulevé en particulier par Monjardet (1996) et Brodeur (2001, p. 319-321) qui discutent la proposition d'Egon Bittner ; voir également Brodeur (2003).

2. En France, Police Nationale et Gendarmerie Nationale sont deux institutions nationales parallèles administrées indépendamment qui exercent, sur des territoires de types différents, les mêmes fonctions de police: ordre public, police routière, police judiciaire, missions de prévention, renseignement, etc. Le territoire français est partagé en zones de compétence, découpées en suivant le maillage communal à l'échelon de chaque département, entre «zone police » (villes moyennes, grandes villes et cœur des métropoles) et «zone gendarmerie » (espaces ruraux, petites villes, espaces périurbains de grandes agglomérations) qui font l'objet de réorganisations récurrentes. De plus, des polices municipales, sous l'entière autorité des maires, ont des responsabilités très limitées en matière d'ordre public, sans aucun pouvoir judiciaire, essentiellement liées à la surveillance et à la prévention. Le terme "police » et l'adjectif " policier» désignent dans ce texte le travail de police en général - ici, celui de la Gendarmerie Nationale.

3. Dans cet article, j'utilise intentionnellement l'anglicisme crime mapping (en italique) pour désigner non pas les cartographies de la criminalité dans leur ensemble et leur diversité, mais le concept particulier développé dans les polices de grandes villes des États-Unis, basé sur l'utilisation de SIG et de bases de données.

4. Le terme de culture professionnelle est employé ici dans un sens général (voir Chan, 2004b) et ne fait pas référence aux débats sur une culture professionnelle spécifique à la police (cop culture) qui est principalement déterminée par son rapport à la violence dans une institution hiérarchisée (discutée entre autres par Monjardet (1994) et Behr (2006)).

5. Au sein des brigades locales (équivalent à une ou plusieurs communes), les gendarmes exercent l'ensemble des fonctions de terrain (police-secours, police judiciaire, prévention, renseignement) ; au niveau de la compagnie (équivalent à un canton), sont regroupées des unités spécialisées (par ex. les Pelotons de Surveillance et d'Intervention de la Gendarmerie - PSIG) ; au niveau du groupement (équivalent à un département), ce sont des brigades spécialisées qui sont en charge des différents domaines (Brigades Recherche - BR pour la police judiciaire, ou Brigade Départementale de Renseignements et d'Investigations Judiciaires - BDRIJ, chargée du travail statistique et de l'information pour la police judiciaire), les services et offices nationaux sont 
quant à eux très spécialisés et disposent de moyens spécifiques d'intervention, de recherche et/ ou de communication.

6. La cartographie embarquée des unités de terrain tout comme les cartographies utilisées dans la gendarmerie mobile, entre autres, n'ont pas fait l'objet de l'enquête, qui n'est pas exhaustive.

7. Les entretiens étaient tantôt centrés autour d'un interlocuteur, tantôt devenaient des discussions semi-collectives, incluant des collègues sollicités ou simplement curieux, de manière très variable. Cela a donné lieu à un discours à plusieurs voix, dont la focale principale changeait, tout comme le lieu de l'entretien, de poste de travail en poste de travail. Dans ces conditions, la majeure partie des entretiens et des séquences de travail n'ont pas fait l'objet d'enregistrement mais de prises de notes. Les démonstrations d'usage étaient des cas exemplaires choisis par les interlocuteurs et selon mes demandes. Les illustrations reproduites ici sont des extraits d'images, tantôt photographiées, tantôt issues de documents électroniques ou de copies d'écrans souvent recadrés.

8. Brodeur distingue très clairement deux traditions de police: «la tradition continentale européenne, caractérisée par un petit nombre de grands appareils policiers hautement centralisés et polyvalents, et la tradition anglo-saxonne d'origine britannique, caractérisée par un plus grand nombre d'appareils de taille relativement modeste et par l'étroitesse relative de leur mandat » (Brodeur, 2003).

9. Le concept de territoire fait débat et ce particulièrement entre deux traditions (voir Antonsich, 2011 ; Debarbieux, 1999) : la discussion anglophone d'une géographie plutôt politique questionne le territoire comme un objet historique intrinsèquement lié à l'État et à la notion de souveraineté - et aussi de sécurité (Elden, 2010 :p. 760). La discussion francophone d'une géographie plutôt sociale questionne le territoire comme modalité de l'appropriation de l'espace, comme relevant de pratiques et représentations partagées voire concurrentes (Aldhuy ,2008 ; Di Méo ,1999 ; Ripoll et Veschambre, 2005). Le concept de «projet territorial» que j'utilise dans ce texte joue sur la combinaison de ces deux significations, en désignant le projet étatique territorial dont la réalisation passe par le travail d'appropriation des acteurs, ici de la gendarmerie.

10. La notion de Raumcontainer (ou containerraum) désigne en allemand une manière de concevoir l'espace : un espace est une entité « contenant » elle-même les phénomènes étudiés (le paysage et son climat ; l'État et son territoire...). Cette notion est surtout utilisée depuis les années 2000 dans le contexte d'approches constructivistes de l'espace pour se distancier d'approches essentialistes ou naturalistes : voir aussi Belina and Miggelbrink (2010) et en français Germes et al. (2011).

11. La «MRT » permet d'identifier alliés et adversaires en situation de crise et dans un lieu précis, d'élaborer et de communiquer une stratégie en fonction d'un objectif.

12. Contrairement à la notion d'espace-container, celle de déterminisme spatial met l'accent sur la recherche de causalité.

13. L'espace est le support d'une série d'objets physiques, caractérisés par leur localisation et leur configuration, et d'une série d'acteurs, caractérisés par leur localisation, leur mouvement et leur intention. La distance entre les acteurs et leur disposition relative sont les deux dimensions principales de cette conception « euclidienne » de l'espace.

14. « praktisch wahr gemachte Abstraktionen », (Belina, 2009). 


\section{RÉSUMÉS}

Le "crime mapping" (ou cartographie de la criminalité), très développé dans les polices métropolitaines ou régionales de pays anglophones, fait l'objet de controverses intenses. Celles-ci sous-estiment cependant deux éléments: premièrement, l'antériorité de pratiques cartographiques dans la police à l'acquisition des outils de cartographie numérique; deuxièmement, des pratiques cartographiques sur le terrain caractérisées par l'autodidactisme. Les pratiques policières de cartographie relèvent ainsi d'une culture professionnelle particulière distincte de l'expertise des cartographes professionnels : cet article met en évidence la dimension vernaculaire du travail cartographique policier et montre en quoi les multiples cartographies policières sont malgré tout au service d'un projet territorial de contrôle. Grâce à une enquête sur la cartographie dans la gendarmerie nationale française, l'article contribue à une approche géographique de la police ancrée dans la cartographie critique et la géographie francophone.

Crime mapping in police work is subject to many controversies, which underestimate two important elements. First, the anteriority of mapping practices within the police before the implementation of crime mapping technologies. Secondly, mapping practices in the police are often self-taught i.e. without proper cartographical education. Thus, police mapping practices have to be unterstood as a particular professionnal culture, and should be distinguished from the expertise of professionnal cartographers. This paper highlights the vernacular dimension of police cartographic work and shows how the multiple police cartographies serve a territorial project aimed at control. Thanks to a field research about cartography in the french Gendarmerie Nationale, the paper contributes to a police geography rooted in international critical cartography and francophone geography.

\section{INDEX}

Mots-clés : Police, France, gendarmerie, pratique cartographique, cartographie critique, crime mapping

Keywords : Police, France, Gendarmerie; mapping practices; critical cartography; crime mapping

\section{AUTEUR}

\section{MELINA GERMES}

Melina Germes, melina.germes@cnrs.fr, est géographe, Chargée de recherche au CNRS, membre de l'UMR 5185 ADESS. Elle a publié récemment :

- Germes M., 2013. Working for the State. A Reading of the French Police Discourses about "banlieues". In Briken, K. und Eick, V., Urban (In)Security: Policing The Neoliberal Crisis, Red Quill Books, Ottawa.

- Glasze G., Pütz R., Germes M., Brailich A. \& Schirmel H., 2012. "The Same but not the Same”. The Discursive Constitution of Large Housing Estates in Germany, France and Poland. Urban Geography (8) 33, p. 1192-1211.

- Germes M., Glasze G. \& Weber F., 2011. « Neue Kulturgeographie » - Débats et perspectives au sein de la nouvelle géographie culturelle germanophone. Cybergeo : European Journal of Geography [online], n 556, 24.10.2011, [http://cybergeo.revues.org/24727]. 